This article is licensed under the Creative Commons Attribution-NonCommercial 4.0 International License (CC BY-NC) (http://www.karger.com/Services/OpenAccessLicense). Usage and distribution for commercial purposes requires written permission.

\title{
Nilotinib-Induced Acute Pancreatitis in a Patient with Chronic Myeloid Leukemia
}

\author{
Vihang Patel Anil Pattisapu Karim Attia John Weiss
}

Mount Carmel Health System, Columbus, OH, USA

\section{Keywords}

Pancreatitis · Chronic myeloid leukemia

\begin{abstract}
Nilotinib, a second-generation tyrosine kinase inhibitor, is used for treatment of chronic myeloid leukemia (CML); it has been widely used especially for imatinib-resistant CML. Despite being a novel drug in this therapeutic class, it has the potential to be harmful. We present the case of an elderly woman who developed life-threatening acute pancreatitis as an adverse event after having started the drug. There is only one reported case in the literature of nilotinib-induced acute pancreatitis. The purpose of this case report is to educate physicians who prescribe this medication to be aware of potential life-threatening adverse events. As more and more therapies are available, physicians should be aware of potential effects of cancer treatment that could be life-threatening to patients.

(c) 2017 The Author(s)

Published by S. Karger AG, Basel
\end{abstract}




\section{Case Reports in Gastroenterology}

\section{Introduction}

Nilotinib (Tasigna) is a second-generation tyrosine kinase inhibitor employed in the treatment of chronic myeloid leukemia (CML). It is a drug that has been utilized by oncologists since 2007. CML is a type of myeloproliferative disorder, thus belonging to a group of disorders that affect the myeloid cell lineage and include polycythemia vera, essential thrombocythemia, and primary myelofibrosis. These are typically caused by an acquired genetic defect of the myeloid stem cells. CML is usually diagnosed incidentally through a complete blood count obtained for other reasons or through recognition of constitutional symptoms like fever, chills, and weight loss combined with splenomegaly. Traditionally, CML is caused by an acquired translocation of chromosomes 9 and 22 [t(9;22)] with the development of a fusion gene known as $B C R-A B L$ that encodes a mutant, activated tyrosine kinase, leading to constant downstream proliferation of the myeloid cell line. Untreated, CML can progress to a transitional accelerated phase or progress to an overt blast crisis, which is an acute leukemia that is usually fatal.

Tyrosine kinase inhibitors target the protein product of the $B C R-A B L$ gene, leading to a decrease in tumor cell proliferation. Imatinib was the first drug developed in this class. Traditionally, tyrosine kinase inhibitors have been associated with pancreatitis; yet, the incidence of acute pancreatitis has been reported to be less than $1 \%$. Nilotinib has been reported to be associated with elevated serum lipase levels [1-3]. However, the literature is scarce on reported cases of nilotinib-induced acute pancreatitis. We here report a case of this adverse event from this novel CML drug [1].

\section{Case Report}

A 66-year-old woman who had been diagnosed with CML 3 months prior to this encounter was treated initially with imatinib but, because of nausea and edema, had to stop therapy and was switched to nilotinib. After a few days of being on nilotinib, she presented to the emergency department at Mount Carmel West Hospital with severe, acute abdominal pain and inability to tolerate oral intake. Her vital signs on presentation showed a temperature of $99.5^{\circ} \mathrm{F}$, a heart rate of $86 \mathrm{bpm}$, a respiratory rate of $20 \mathrm{BRPM}$, and a blood pressure of $147 / 60 \mathrm{~mm} \mathrm{Hg}$. The remainder of her physical examination revealed a soft abdomen with moderate midepigastric tenderness to palpation. Serum lipase was elevated at $81 \mathrm{U} / \mathrm{L}$ (normal 18-58). BUN (blood urea nitrogen) was elevated at $19 \mathrm{mg} / \mathrm{dL}$. A CT scan of the abdomen with contrast showed stranding around the head of the pancreas in addition to splenic granulomata and enlarged retroperitoneal lymph nodes (Fig. 1). Thus, the patient had clinical, biochemical, and imaging findings consistent with acute pancreatitis.

The patient had no history of alcohol use. Her lipid panel was unremarkable, with normal triglyceride levels at $60 \mathrm{mg} / \mathrm{dL}$. She did not have any acute biliary abnormalities on magnetic resonance cholangiopancreatography. A review of her current medication list did not reveal any other medications that could potentially cause acute pancreatitis. All other common causes of acute pancreatitis were ruled out. Given that initiation of nilotinib coincided with this presentation of acute pancreatitis, which could not be otherwise explained, it was concluded that the patient had acute pancreatitis secondary to nilotinib. The patient 
was admitted and treated supportively with IV fluids and pain management. Nilotinib was discontinued after a discussion with her oncologist. The patient felt better within 3 days. The lipase level went down to $19 \mathrm{U} / \mathrm{L}$ and BUN down to $4 \mathrm{mg} / \mathrm{dL}$. She was eventually discharged home in a stable condition. Her oncologist switched her to bosutinib (Bosulif), which has not been shown to be associated with pancreatitis.

\section{Discussion}

Nilotinib was first approved by the FDA in October 2007. It is usually reserved for patients that are imatinib resistant. Patients receiving imatinib have been reported to have an up to $90 \%$ hematologic response. However, there is a group of patients with a point mutation in $B C R-A B L$ who do not respond to imatinib. Nilotinib has been shown to respond to 32 of the 33 point mutations in the $B C R-A B L$ gene $[4,5]$. A patient was started on nilotinib and a few days after presented with acute abdominal pain and was found to have acute pancreatitis from nilotinib. All tyrosine kinase inhibitors are known to cause elevation in serum lipase. Up to $20 \%$ of the patients receiving nilotinib have been reported to have isolated elevations in serum lipase [3]. According to a systematic review, about $4 \%$ of patients receiving tyrosine kinase inhibitors have been reported to develop pancreatitis. However, the reported rate of acute pancreatitis among patients on nilotinib is about 1\% (Table 1) [2]. Although the exact mechanism for pancreatitis is unclear, there are a few proposed hypotheses regarding the increase in serum pancreatic enzyme levels. The $c$-abl protein interacts with signaling proteins, nucleocytoplasmic shuttling, and DNA- and actin-binding sites. Therefore, it is possible that $\mathrm{c}-\mathrm{abl}$ inhibition might interfere with the molecular mechanisms regulating pancreatic cell death, inducing pancreatic damage. It may also act on unknown intracellular pathways involved in calcium release from intracellular acinar stores, which regulate exocrine pancreatic secretion, or may promote the accumulation of fatty acid inside the pancreatic acinar cells, which disturbs exocytosis [2].

We decided to present this case, since nilotinib has been utilized more frequently for the treatment of CML. More patients on nilotinib might potentially experience pancreatitis. As newer agents are being utilized more frequently, it is vital for clinicians to monitor patients closely after starting new drugs. This is especially true about medications that have the potential to cause life-threatening adverse reactions.

\section{Statement of Ethics}

After discussing with the patient about reporting our findings on her case for publication, the patient provided consent to publication.

\section{Disclosure Statement}

None of the authors listed have any conflict of interest. 


\section{References}

1 Engel T, Justo D, Amitai M, Volchek Y, Mayan H: Nilotinib-associated acute pancreatitis. Ann Pharmacother 2013;47:e3.

2 Pezzilli R, Corinaldesi R, Morselli-Labate AM: Tyrosine kinase inhibitors and acute pancreatitis. JOP 2010;11:291-293.

-3 Rosti G, Palandri F, Castagnetti F, Breccia M, Levato L, Gugliotta G, et al: Nilotinib for the frontline treatment of $\mathrm{Ph}^{+}$chronic myeloid leukemia. Blood 2009;114:4933-4938.

4 Deremer DL, Ustun C, Natarajan K: Nilotinib: a second-generation tyrosine kinase inhibitor for the treatment of chronic myelogenous leukemia. Clin Ther 2008;30:1956-1975.

-5 Kantarjian H, Giles F, Wunderle L, Bhalla K, O’Brien S, Wassmann B, et al: Nilotinib in imatinib-resistant CML and Philadelphia chromosome-positive ALL. N Engl J Med 2006;354:2542-2551.

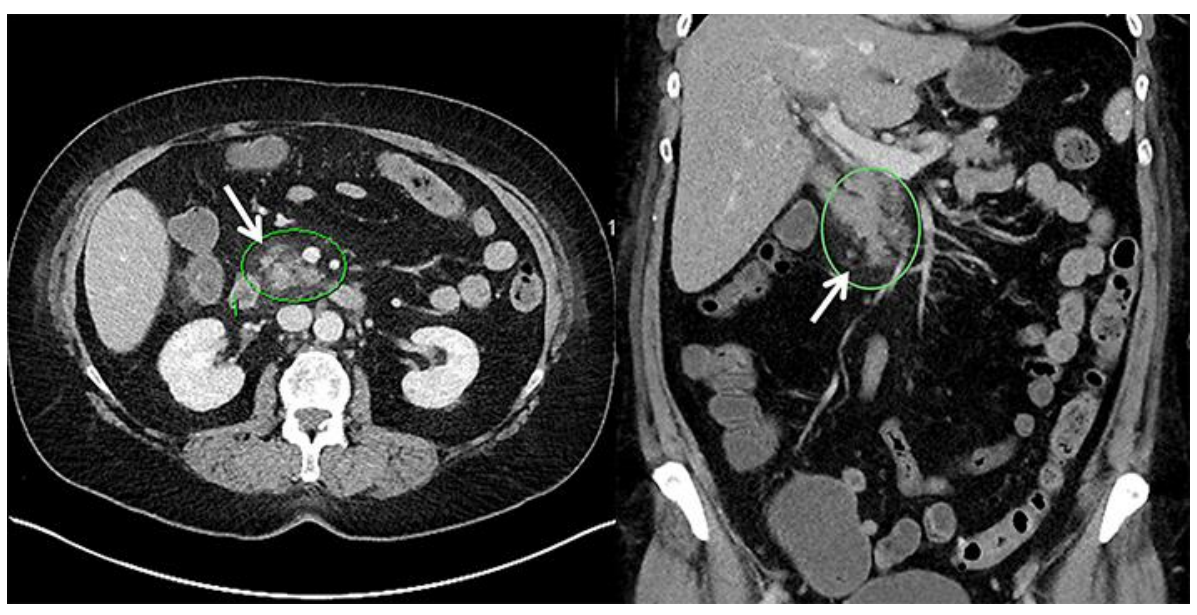

Fig. 1. CT images of the patient, with inflammation around the head of the pancreas (arrows).

Table 1. Frequency of acute pancreatitis among patients treated with nilotinib [2]

\begin{tabular}{lllll}
\hline First author & Year & Disease & Patients, $n$ & Acute pancreatitis, $n(\%)$ \\
\hline Kantarjian & 2007 & CML & 280 & $3(1.1)$ \\
Le Coutre & 2008 & CML & 119 & $1(0.8)$ \\
Hazarika & 2008 & CML & 438 & 0 \\
Rosti & 2009 & CML & 73 & 0 \\
Zhou & 2009 & CML & 23 & 0 \\
Tojo & 2009 & CML & 34 & 0 \\
\hline
\end{tabular}

